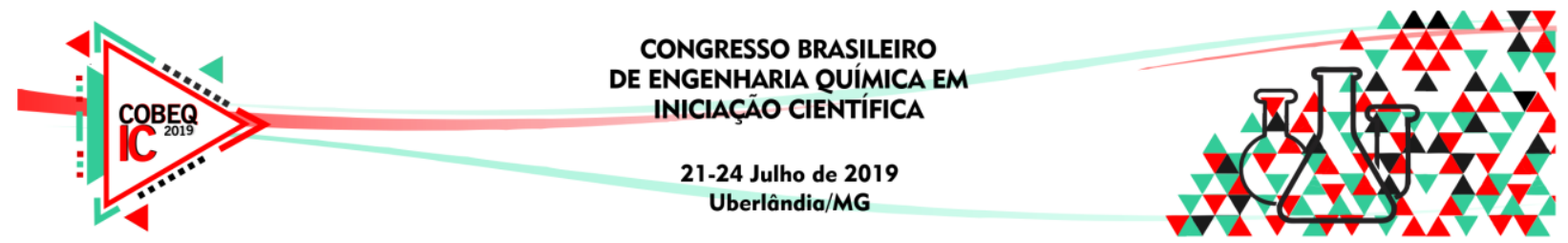

\title{
AVALIAÇÃO DA CONTRIBUIÇÃO DE CARGAS DIFUSAS NA MICROBACIA DO RIO DAS MORTES UTILIZANDO MODELOS MATEMÁTICOS DE CORRELAÇÃO USO DO SOLO E QUALIDADE DE ÁGUA
}

\author{
E. B. MARRA ${ }^{1}$, J. A. M. JUNIOR ${ }^{2}$, M. O. A. F. AGUIAR ${ }^{3}$, O. S. PEREIRA ${ }^{4}$ e C. S. S. \\ PEREIRA $^{2}$ \\ ${ }^{1}$ Universidade de Vassouras, Curso de Engenharia Química \\ ${ }^{2}$ Universidade de Vassouras, Mestrado Profissional em Ciências Ambientais \\ ${ }^{3}$ Universidade de Vassouras, Engenheiro Civil \\ ${ }^{4}$ Universidade Federal Rural do Rio de Janeiro, Departamento de Matemática \\ E-mail para contato: elisabmarra@gmail.com
}

\begin{abstract}
RESUMO - O Rio das Mortes, situado na cidade de Vassouras-RJ e afluente do Rio Paraíba do Sul, recebe diariamente uma alta carga de despejos sem tratamento em toda sua extensão, sendo estas cargas provenientes principalmente de fontes difusas. Para avaliação da contribuição destas cargas pode ser utilizado o Modelo Matemático de Correlação Uso do Solo e Qualidade da Água (MQUAL) que permite mensurar a geração e afluência de cargas difusas e pontuais de poluentes em uma bacia hidrográfica. Para avaliar as cargas difusas lançadas na bacia do Rio das Mortes, realizou-se o presente estudo para estimativa dos parâmetros: fósforo, nitrogênio, demanda bioquímica de oxigênio, sólidos em suspensão e coliformes totais utilizando os modelos matemáticos de correlação e uso do solo e qualidade de água MQUAL 2.0 e MQUAL 1.0. Os resultados indicam um aporte diário de fósforo $(1,017 \mathrm{~kg}$ e 2,481 kg), nitrogênio $(9,175$ e 44,525 kg), demanda bioquímica de oxigênio $(37,743 \mathrm{~kg}$ e $120,877 \mathrm{~kg})$, sólidos em suspensão $(283,104 \mathrm{~kg}$ e $1.931,200 \mathrm{~kg})$ e coliformes totais $(3,97 \mathrm{E}+04 \mathrm{~kg}$ e $1,17 \mathrm{E}+11 \mathrm{~kg})$, para as versões do modelo 2.0 e 1.0, respectivamente, Os resultados obtidos são importantes para a microbacia estudada, pois servem como estimativa inicial para posterior comparação com o estudo de monitoramento do Rio a partir das análises químicas dos parâmetros estimados.
\end{abstract}

\section{INTRODUÇÃO}

De acordo com Bione et al. (2009) o crescimento da população em todo o mundo, tem como consequência a ampliação da demanda de água, principalmente para uso doméstico, industrial e agrícola. Sendo assim, a tendência é de cada vez mais existir a exploração dos recursos hídricos, muitas vezes comprometendo a qualidade da água dos rios por conta do aumento de atividades que se utilizam do próprio corpo hídrico para o despejo de resíduos.

Segundo Mansor et al. (2006), a poluição de um determinado rio pode ser verificada analisando as cargas de poluição lançadas ao mesmo, classificadas em cargas pontuais e cargas difusas. As cargas pontuais são constituídas pelos despejos de efluentes domésticos ou 


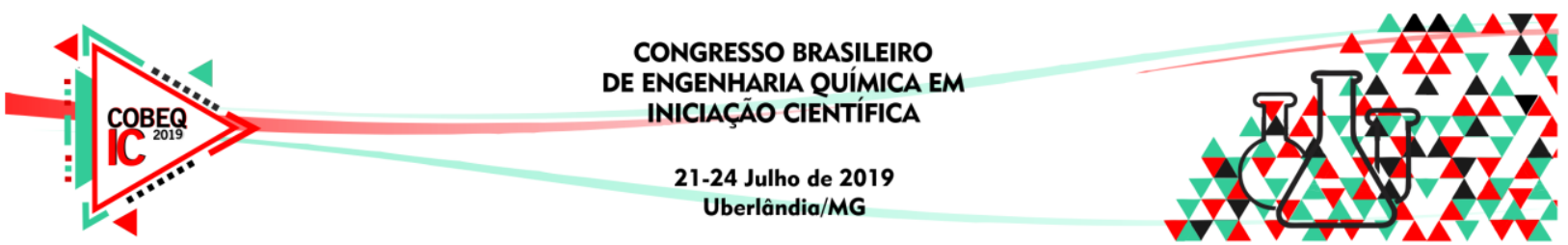

industriais, e as difusas, são aquelas geradas em áreas extensas e que, associadas às precipitações pluviométricas, chegam às águas superficiais de forma intermitente.

Dentre os parâmetros vinculados às cargas difusas, Von Sperling (2005) destaca os parâmetros a serem analisados: nitrogênio e fósforo, demanda bioquímica de oxigênio (DBO), sólidos em suspensão e coliformes totais. Nitrogênio e fósforo são nutrientes importantes para a estabilização da matéria orgânica, auxiliando no crescimento de microrganismos responsáveis por esta tarefa. Já a DBO é um dos parâmetros mais importantes na caracterização do nível de poluição de um corpo hídrico, tendo em vista que a matéria orgânica tem a função de consumir o oxigênio dissolvido na água, retratando indiretamente o teor de matéria orgânica nos efluentes. No caso de sólidos em suspensão, podem ser sedimentáveis ou não, onde geralmente são compostos por matéria orgânica. Os coliformes totais compreendem um grande número de bactérias isoladas de amostras de água e solos não necessariamente poluídos, sendo encontrados também em fezes de animais de sangue quente.

O monitoramento de fontes difusas de nutrientes na escala de uma bacia hidrográfica envolve muitas incertezas devido ao fato desta poluição ser de difícil quantificação, uma vez que depende da interação de diferentes fatores, como a intensidade e duração das precipitações, o tipo e as formas de uso dos solos. No Brasil, a disponibilidade de dados de monitoramento de fontes poluidoras difusas para as bacias hidrográficas é escassa.

De acordo com Straskraba e Tundisi (2008) e Moruzzi et al (2012) no gerenciamento da qualidade da água os modelos matemáticos são amplamente utilizados e podem ser usados para estimar os focos de poluição existente nas bacias hidrográficas, fornecendo estimativas que permitam a tomada de decisões sobre diferentes opções de gerenciamento e apoiar decisões voltadas ao planejamento ambiental em curto, médio e longo prazo.

Dentre os modelos matemáticos citados na literatura destacam-se os modelos matemáticos de correlação e uso do solo e qualidade de água (MQUAL). De acordo com o Relatório do Reservatório de Billings, feito pela Secretaria do Estado do Meio Ambiente - SP (2010), o modelo (MQUAL) possuí várias versões e os que mais se destacam são: MQUAL 1.0 (1997) e MQUAL 2.0 (1998).

Portanto, diante da necessidade do conhecimento das cargas difusas que aportam ao Rio das Mortes, o presente trabalho avaliou essa contribuição através da aplicação do modelo matemático MQUAL nas versões 2.0 e 1.0 (SMA, 2010) que se baseia na área de uso e cobertura do solo a fim de estimar os valores de contribuição para os indicadores: fósforo total, nitrogênio total, DBO, sólidos em suspensão e coliformes totais, que servirão como base para posteriores estudos da capacidade de autodepuração de corpos hídricos.

\section{MATERIAIS E MÉTODOS}

A microbacia do Rio das Mortes, localizada na cidade de Vassouras/RJ é ilustrada conforme Figura 1, evidenciando em azul a demarcação da extensão do trecho estudado. Para a produção desta imagem utilizou-se uma aeronave remotamente pilotada (ARP) DJI Mavic Pro com precisão de 0,41 metros associado ao aplicativo Drone deploy para o planejamento e execução dos planos de voo com uma altitude de 120 metros acima do solo com sobreposição frontal de $75 \%$ e lateral de $65 \%$. 


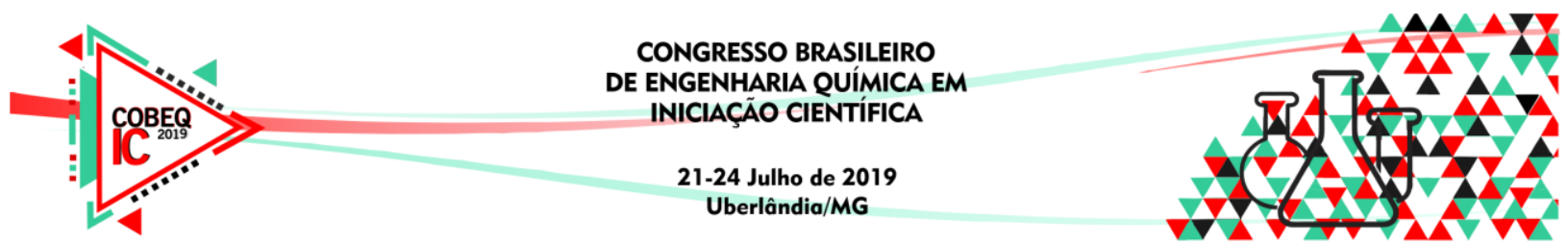

Figura 1. Área de estudo da microbacia

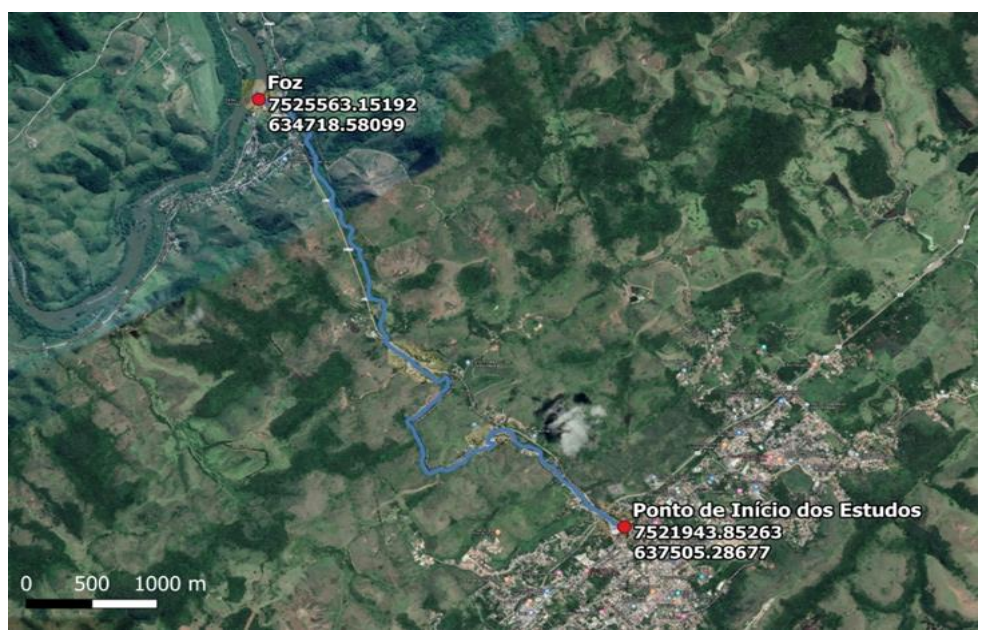

A bacia hidrográfica do Rio Paraíba do Sul está compreendida em três Estados da Região Sudeste (São Paulo, Rio de Janeiro e Minas Gerais). Um ponto em destaque é sua rede de drenagem, que ocupa uma área de $57.000 \mathrm{~km}^{2}$, somente no Estado do Rio de Janeiro, a área de ocupação deste corpo hídrico chega a $22.600 \mathrm{~km}^{2}$, evidenciando assim, a extrema importância deste rio (Bione, 2009). Já a microbacia do Rio das Mortes, afluente do Rio Paraíba do Sul, apresenta uma microbacia com uma área de drenagem de 49,66 km² incluindo: atividade agrícola, reflorestamento, pastagens, área florestal e área urbana (Atlas CBH-MPS, 2017).

\subsection{Avaliação do aporte das cargas difusas}

Avaliou-se as cargas difusas de fósforo total, nitrogênio total, demanda bioquímica de oxigênio (DBO), sólidos em suspensão e coliformes totais utilizando o modelo matemático (MQUAL) em suas versões 2.0 e 1.0, considerando os coeficientes de exportação relacionados ao uso e ocupação do solo. Os coeficientes de exportação são valores tabelados provenientes de estudos de cargas geradas em relação ao uso do solo na bacia de origem. Os valores dos coeficientes de exportação determinados pela Secretaria Estadual de Meio Ambiente (SMA, 2003) e considerados nos cálculos do presente estudo são apresentados na Tabela 1 e 2 para cada uma das categorias de uso e ocupação do solo.

Tabela 1. Coeficientes de exportação obtidos do banco de dados do MQUAL 2.0

\begin{tabular}{|c|c|c|c|c|c|}
\hline \multirow{2}{*}{ Uso e cobertura do solo } & $\begin{array}{c}\text { Fósforo } \\
\text { Total }\end{array}$ & $\begin{array}{c}\text { Nitrogênio } \\
\text { Total }\end{array}$ & DBO & $\begin{array}{c}\text { Sólidos em } \\
\text { Suspensão }\end{array}$ & $\begin{array}{c}\text { Coliformes } \\
\text { totais }\end{array}$ \\
\cline { 2 - 6 } & \multicolumn{5}{|c|}{$\mathbf{k g / \mathbf { k m } ^ { 2 } \text { .dia }}$} \\
\hline \hline Atividade Agrícola & 0,066 & 0,227 & 0,933 & 10,455 & $1,00 \mathrm{E}+04$ \\
\hline Reflorestamento & 0,002 & 0,060 & 0,247 & 2,500 & $1,00 \mathrm{E}+02$ \\
\hline Pastagem & 0,005 & 0,090 & 0,370 & 8,000 & $1,00 \mathrm{E}+03$ \\
\hline Mata/Capoeirão/Mata galeria & 0,002 & 0,060 & 0,247 & 2,500 & $1,00 \mathrm{E}+02$ \\
\hline Áreas Urbanas & 0,136 & 0,951 & 3,913 & 0,550 & $1,00 \mathrm{E}+03$ \\
\hline
\end{tabular}

Fonte: Adaptada - SMA SP (2010). 


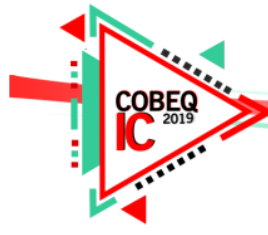

Tabela 2. Coeficientes de exportação obtidos do banco de dados do MQUAL 1.0

\begin{tabular}{|c|c|c|c|c|c|}
\hline $\begin{array}{c}\text { Uso e cobertura do } \\
\text { solo }\end{array}$ & Fósforo Total & $\begin{array}{c}\text { Nitrogênio } \\
\text { Total }\end{array}$ & DBO & $\begin{array}{c}\text { Sólidos em } \\
\text { Suspensão }\end{array}$ & $\begin{array}{c}\text { Coliformes } \\
\text { Totais }\end{array}$ \\
\cline { 2 - 6 } & \multicolumn{5}{|c|}{$\mathbf{k g} / \mathbf{k m}$.dia } \\
\hline \hline Atividade Agrícola & 0,346 & 2,950 & 7,315 & 230,000 & $1,00 \mathrm{E}+11$ \\
\hline Reflorestamento & 0,039 & 0,600 & 1,197 & 20,000 & $1,00 \mathrm{E}+08$ \\
\hline Pastagem & 0,050 & 0,900 & 2,250 & 40,000 & $1,00 \mathrm{E}+09$ \\
\hline $\begin{array}{c}\text { Mata/Capoeirão/ } \\
\text { Mata galeria }\end{array}$ & 0,039 & 0,600 & 1,197 & 20,000 & $1,00 \mathrm{E}+08$ \\
\hline Áreas Urbanas & 0,034 & 1,274 & 5,535 & 50,000 & $1,00 \mathrm{E}+09$ \\
\hline
\end{tabular}

Fonte: Adaptada - SMA SP (2010).

Para os cálculos de cargas difusas a partir da Equação 1, uma associação com o uso e cobertura do solo deve ser feita.

$$
C D=A \cdot C E
$$

Onde:

$\mathrm{CD}=$ carga difusa $(\mathrm{kg} / \mathrm{dia})$

$\mathrm{A}=$ Área total de cada classe $\left(\mathrm{km}^{2}\right)$

$\mathrm{CE}=$ Coeficiente de exportação $\left[\left(\mathrm{kg} /\left(\mathrm{km}^{2}\right.\right.\right.$.dia $\left.)\right]$

Para o desenvolvimento dos cálculos para a modelagem utilizou-se o Atlas das Bacias Hidrográficas da Região do Médio Paraíba do Sul, com as principais atividades e uso do solo para a microbacia estudada, conforme evidenciado na Tabela 3.

Tabela 3. Áreas das classes do uso e cobertura do solo da Microbacia do Rio das Mortes

\begin{tabular}{|c|c|}
\hline Uso e cobertura do solo & Área $\left(\mathbf{k m}^{\mathbf{2}}\right)$ \\
\hline Atividade Agrícola & 0,80 \\
\hline Reflorestamento & 0,96 \\
\hline Pastagem & 29,80 \\
\hline Mata/Capoeirão/Mata galeria & 12,30 \\
\hline Áreas Urbanas & 5,80 \\
\hline
\end{tabular}

Fonte: Adaptada Atlas CBH-MPS (2017).

O modelo MQUAL 2.0 considera cargas médias anuais geradas em tempo úmido e seco, enquanto que a versão 1.0 utiliza-se cargas somente em tempo seco. No presente trabalho avaliaram-se as contribuições dos parâmetros considerando tempo seco. As equações utilizadas no modelo MQUAL, foram inseridas no software Microsoft Excel ${ }^{\circledR}$.

\section{RESULTADOS E DISCUSSÕES}

Os resultados estimados para a contribuição das cargas difusas na microbacia do Rio das Mortes totais são apresentados na Tabela 4. 

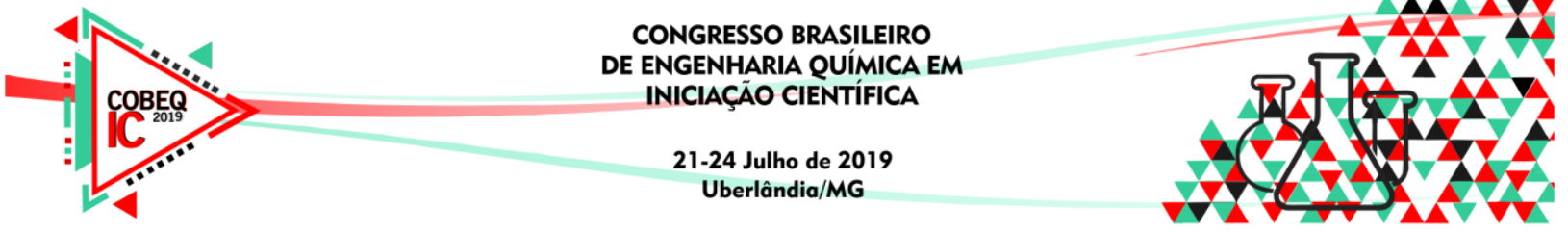

Tabela 4. Simulação de cargas difusas (kg/dia) geradas na microbacia do Rio das Mortes

\begin{tabular}{|c|c|c|c|c|c|}
\hline \multicolumn{6}{|c|}{ MQUAL 2.0 } \\
\hline \multirow{2}{*}{$\begin{array}{c}\text { Uso e cobertura do } \\
\text { solo }\end{array}$} & $\begin{array}{c}\text { Fósforo } \\
\text { Total }\end{array}$ & $\begin{array}{c}\text { Nitrogênio } \\
\text { Total }\end{array}$ & DBO & $\begin{array}{l}\text { Sólidos em } \\
\text { Suspensão }\end{array}$ & Coliformes totais \\
\hline & \multicolumn{5}{|c|}{$\mathrm{kg} / \mathrm{dia}$} \\
\hline Atividade Agrícola & 0,053 & 0,182 & 0,746 & 8,364 & $8,00 \mathrm{E}+03$ \\
\hline Reflorestamento & 0,002 & 0,058 & 0,237 & 2,400 & $9,60 \mathrm{E}+01$ \\
\hline Pastagem & 0,149 & 2,682 & 11,026 & 238,400 & $2,98 \mathrm{E}+04$ \\
\hline $\begin{array}{l}\text { Mata/Capoeirão/Mata } \\
\text { galeria }\end{array}$ & 0,025 & 0,738 & 3,038 & 30,750 & $1,23 \mathrm{E}+03$ \\
\hline Áreas Urbanas & 0,789 & 5,516 & 22,695 & 3,190 & $5,80 \mathrm{E}+02$ \\
\hline Total & $\underline{1,017}$ & $\underline{9,175}$ & $\underline{37,743}$ & 283,104 & $\underline{3,97 E+04}$ \\
\hline \multicolumn{6}{|c|}{ MQUAL 1.0 } \\
\hline $\begin{array}{c}\text { Uso e cobertura do } \\
\text { solo }\end{array}$ & \multicolumn{5}{|c|}{$\mathrm{kg} / \mathrm{dia}$} \\
\hline Atividade Agrícola & 0,277 & 2,360 & 5,852 & 184,000 & $8,00 \mathrm{E}+10$ \\
\hline Reflorestamento & 0,037 & 0,576 & 1.149 & 19,200 & $9,60 \mathrm{E}+07$ \\
\hline Pastagem & 1,490 & 26,820 & 67,050 & $1.192,000$ & $2,98 \mathrm{E}+10$ \\
\hline $\begin{array}{c}\text { Mata/Capoeirão/Mata } \\
\text { galeria }\end{array}$ & 0,480 & 7,380 & 14,723 & 246,000 & $1,23 \mathrm{E}+09$ \\
\hline Áreas Urbanas & 0,197 & 7,389 & 32,103 & 290,000 & $5,80 \mathrm{E}+09$ \\
\hline Total & 2,481 & 44,525 & 120,877 & $1.931,200$ & $1,17 \mathrm{E}+11$ \\
\hline
\end{tabular}

Ao realizar uma análise inicial dos valores de área apresentados na Tabela 3, observa-se o maior valor voltado para pastagem, e o menor valor para atividade agrícola.

De acordo com os dados apresentados na Tabela 4 observam-se resultados elevados para o uso do solo em área urbana e pastagem. Na versão 2.0 e na versão 1.0 o uso para pastagens compreende valores mais altos do que em área urbana por exemplo. Para os outros parâmetros, observa-se uma proporcionalidade entre eles, apesar dos valores serem bem mais elevados na versão 1.0 .

A principal diferença entre as duas versões é baseada nos coeficientes de exportação, pois na versão 2.0 houve uma atualização com a inserção de novos dados sobre cargas difusas, ocorrendo uma redução para os padrões rurais de uso do solo e um acréscimo para os padrões de uso urbanos e para as cargas de origem doméstica. Ressalta-se ainda, que a diferença na geração dos coeficientes de exportação de cada versão pode ser atribuída às análises realizadas durante cada estudo, em locais e quantidade de microbacias diferentes para o MQUAL $1.0 \mathrm{e}$ 2.0 .

Para os parâmetros fósforo total, nitrogênio total e DBO, os resultados expressam a realidade uma vez que ocorre lançamento de esgoto sem tratamento no corpo hídrico. $\mathrm{O}$ uso inadequado de pastagens, principalmente nas margens do rio, também corrobora os resultados obtidos.

Ainda que os modelos matemáticos sejam fundamentais neste tipo de estudo, e conforme citado por Gonçalves (2009) dados obtidos em campo são necessários para calibração do modelo. 


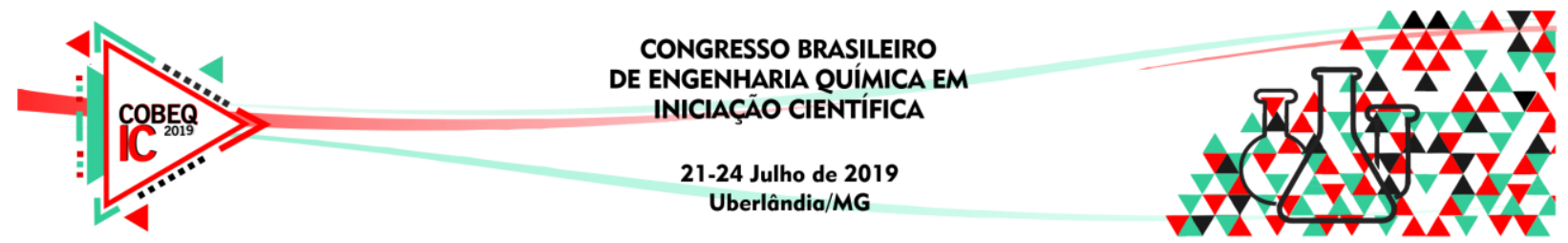

\section{CONCLUSÃO}

Apesar dos modelos matemáticos serem uma ferramenta de grande importância para a estimativa de cargas difusas, trabalhos de modelagem e simulação de autodepuração considerando o lançamento de cargas difusas correlacionadas ao uso do solo e qualidades da água são poucos no estado do Rio de Janeiro. Os resultados obtidos servem como estimativa inicial para posterior comparação com o estudo de monitoramento a partir das análises químicas dos parâmetros estimados.

\section{REFERÊNCIAS}

BIONE, M. A. A.; DANTAS, R. D. L.; TAVARES, R. G.; ALBUQUERQUE, C. D.; SOARES, T.; SILVA, E. D. F. Poluição do Rio Capibaribe por esgoto doméstico. In: IX Jornada de Ensino, Pesquisa e Extensão, 2009.

CBH - MPS, Atlas das Bacias Hidrográficas da Região do Médio Paraíba do Sul, 2017.

GONÇALVES, J.C.S.I. Desenvolvimento de modelo numérico para a simulação da qualidade da água em rios utilizando o software Vensin PLE®. São Carlos, 2009. 203 p. Dissertação (de Mestrado) - Escola de Engenharia de São Carlos, Universidade de São Paulo.

MANSOR, M. T.C.; FILHO, J. T.; ROSTON, D. M. Avaliação preliminar das cargas difusas de origem rural, em uma sub-bacia do Rio Jaguari, SP. Revista Brasileira de Engenharia Agrícola e Ambiental, 2006.

MORUZZI, R. B.; CONCEIÇÃO, F. T.; SOUZA, S. D.; HONDA, F. P.; NAVARRO, G. R. B. Avaliação de cargas difusas e simulação de autodepuração no córrego da Água Branca, Itirapina (SP). Geociências (São Paulo), v. 31, n. 3, p. 447-458, 2012.

SANTOS, A. F.; KOBIYAMA, M. Contribuição potencial de cargas poluentes na bacia do Rio das Pedras, no município de Guarapuava-PR. RECEN-Revista Ciências Exatas e Naturais, v. 5, n. 1, p. 33-46, 2003.

SMA. Secretaria Estadual do Meio Ambiente. Elaboração do Plano de Desenvolvimento e Proteção Ambiental da Bacia Hidrográfica do Reservatório Billings, 2010. Disponível em: 〈http://pdpa.cobrape.com.br/Arquivos/Pdpas/PDPA-Billings.pdf>. Acesso em: $1 \mathrm{fev}$ 2019.

STRASKRABA, M.; TUNDISI, J.G. Diretrizes para o gerenciamento de lagos: gerenciamento da qualidade da água em represas. $2^{a}$ ed. São Carlos: Cubo Multimídia, 300 p., 2008.

VON SPERLING, M. Introdução à qualidade das águas e ao tratamento de esgotos. Editora UFMG, 2005. 